\title{
Precise Construction of Cell-instructive 3D Microenvironments by Photopatterning a Biodegradable Hydrogel
}

Zhengwei Cai, ${ }^{\dagger}$ Kaiping Huang, ${ }^{\dagger}$ Chunyan Bao, ${ }^{* \dagger}{ }^{\dagger}$ Xuebin Wang, ${ }^{\dagger}$ Xiangchao Sun, ${ }^{\ddagger}$ Hong Xia, ${ }^{\dagger}$ Qiuning Lin,${ }^{\dagger}$ Yi Yang, ${ }^{\dagger}$ Linyong $\mathrm{Zhu}^{*}, \dagger$

'Optogenetics \& Synthetic Biology Interdisciplinary Research Center, State Key

Laboratory of Bioreactor Engineering, East China University of Science and

Technology, 130\# Meilong Road, Shanghai 200237, China.

State Key Laboratory of Integrated Optoelectric, College of Electronic Science \& Engineering, Jilin University, Changchun, 130012, Jilin, China.

\section{Materials and Characterizations}

\subsection{Materials}

All chemical reagents were purchased from commercial available sources such as Sigma and Aladdin. Deionized water was used to prepare all aqueous systems. Unless specifically mentioned, the buffer used in the experiments is $0.01 \mathrm{M}$ phosphate buffer saline (PBS, $\mathrm{pH}=$ 7.2). Matrix metalloproteinase substrate peptide AcGCRDGPQGIWGQDRCG-NH,$\quad$ cyclo(Arg-Gly-Asp-d-Phe-Lys) （c(RGDfK)), cyclo(Arg-Gly-Asp-d-Phe-Cys) (c(RGDfC)) were purchased from Apeptide Co., Ltd., Shanghai, China. Dulbecco's modified eagle's medium (DMEM, Gibco), Live/Dead imaging kit (Invitrogen), Alexa Fluor 488-phalloidin, and 4',6-diamidino-2-phenylindole (DAPI) were purchased from Thermo Fisher Scientific. NB-1, bovium serum albumin (BSA), fetal bovine serum (FBS) and BOC-Lys are commercial available and purchased from Sigma.

\subsection{Characterizations}

Proton spectra ( ${ }^{1} \mathrm{H}$ NMR) were recorded on a Bruker Avance $(600 \mathrm{MHz})$ spectrometer. Chemical shifts were reported in parts per million (ppm) downfield from the $\mathrm{Me}_{4} \mathrm{Si}$ resonance which was used as the internal standard when recording ${ }^{1} \mathrm{H}$ NMR spectra. Absorption spectra were recorded on a Shimadzu UV-2550 UV-Vis spectrometer. Attenuated total reflectance FTIR (ATR-FTIR) spectra were recorded on a NICOLET 5700 
FT-IR spectrometer. Mechanical measurements were recorded on a HAAKE MARS III rheometer. The confocal fluorescence images were performed on a confocal laser scanning microscope (CLSM, Leica-TCS-SP8) with $5 \times, 10 \times$ and $40 \times$ objective lens.

\section{Synthesis and Characterization of HA-NB-VS}

\subsection{Synthesis of HA-NB-VS}

HA
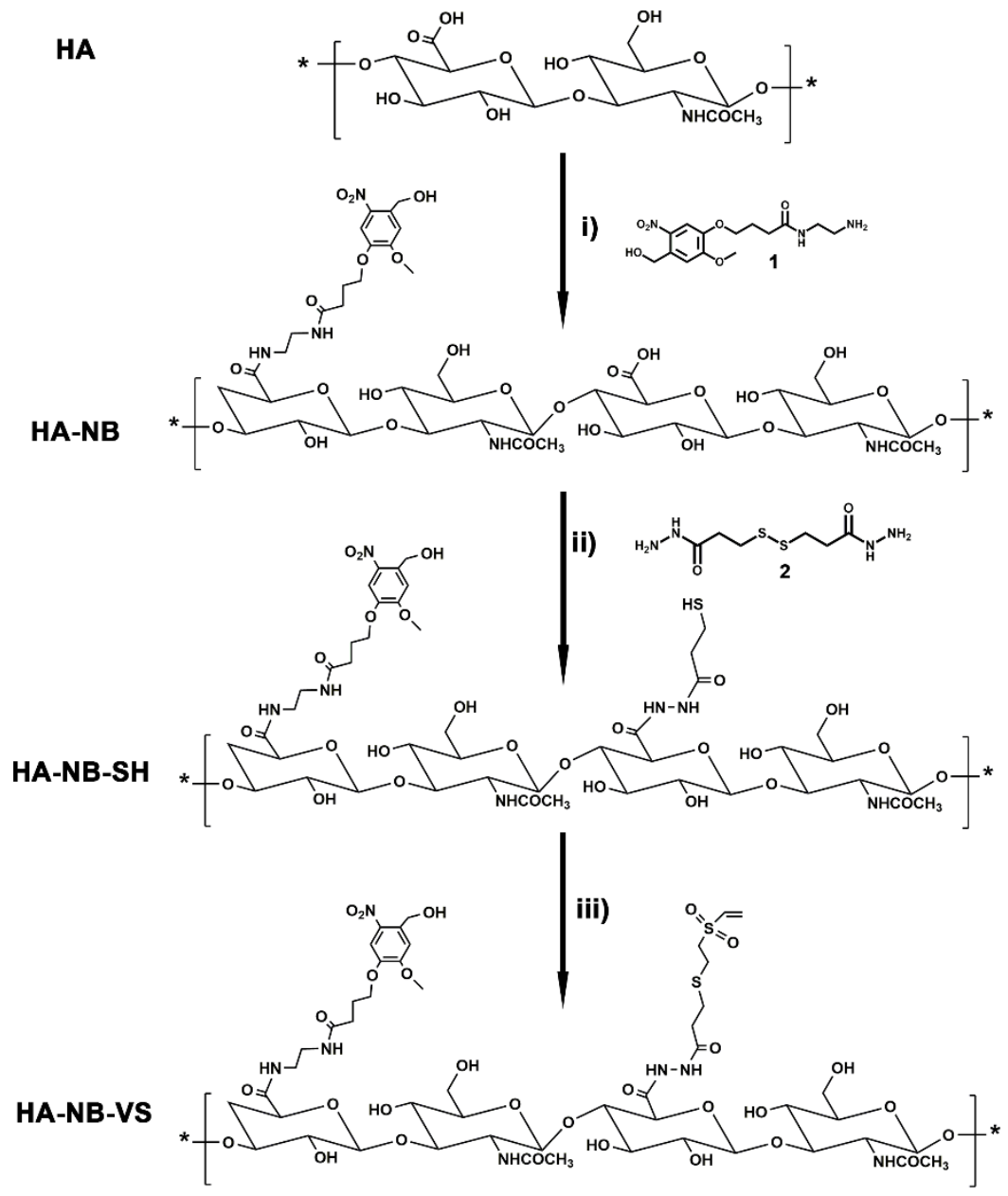

Figure S1. The synthetic procedure for the HA derivatives, including HA-NB, HANB-SH, and HA-NB-VS. The reaction conditions: i) 1, Dimethoxy-1,3,5-triazin-2-yl)4-methyl morpholinium chloride (DMTMM), DMSO/MES buffer (10.0 mM, pH = 5.5), and RT for $12 \mathrm{~h}$; ii) 2, DMTMM, MES buffer $(\mathrm{pH}=5.5)$, and RT for $12 \mathrm{~h}$, then Tris(2carboxyethyl) phosphine) hydrochloride (TCEP-HCl) and RT for 3h; iii) divinyl sulfone, triethanolamine buffer (TEOA, $300 \mathrm{mM}, \mathrm{pH}=8.0$ ) and $\mathrm{RT}$ for $10 \mathrm{~h}$.

\subsection{Characterization of $\mathrm{HA}-\mathrm{NB}$}




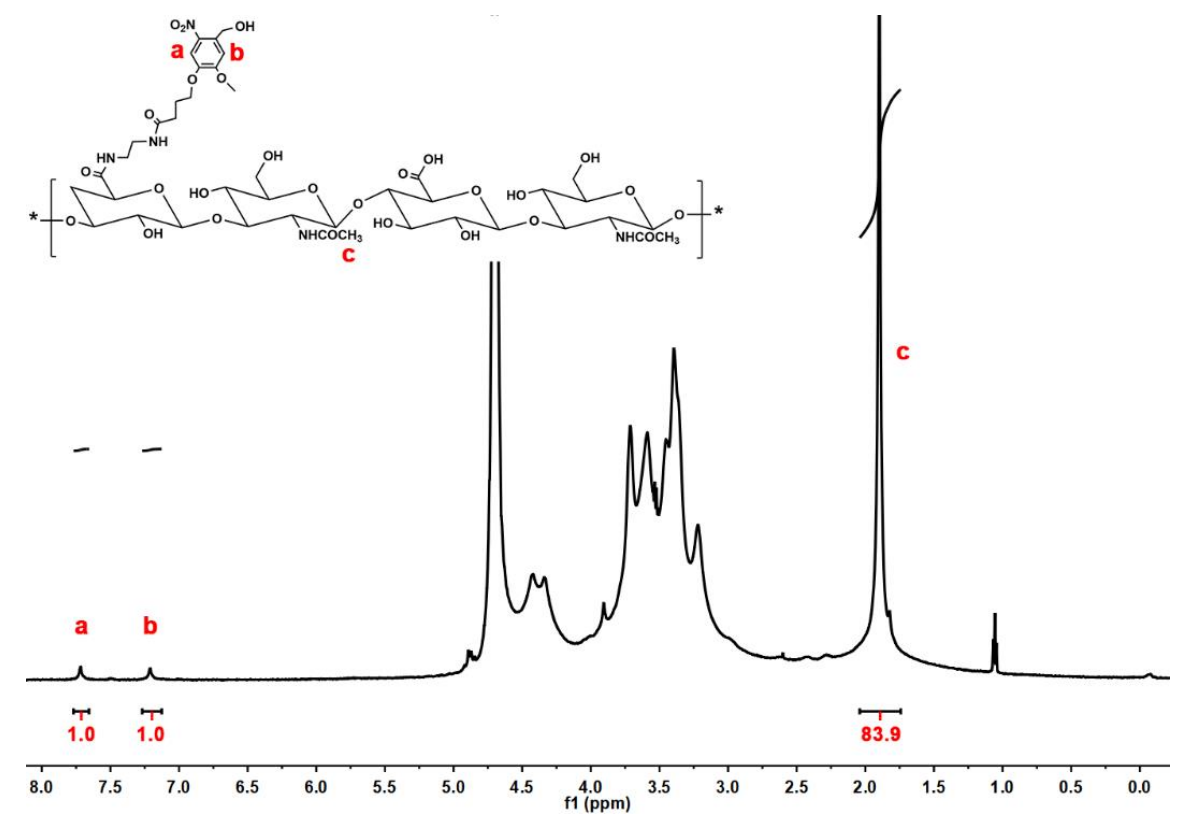

Figure S2. ${ }^{1} \mathrm{H}$ NMR spectrum $\left(600 \mathrm{MHz}, \mathrm{D}_{2} \mathrm{O}\right)$ of $\mathbf{H A}-\mathbf{N B}$.

The substitution degree was calculated by the NMR integral analysis using the following formula $\mathrm{Y} \%=\mathrm{I}_{[\mathrm{a}]} /\left(\mathrm{I}_{[\mathrm{c}]} \div 3\right) \times 100 \%$.

\subsection{Characterization of HA-NB-VS}

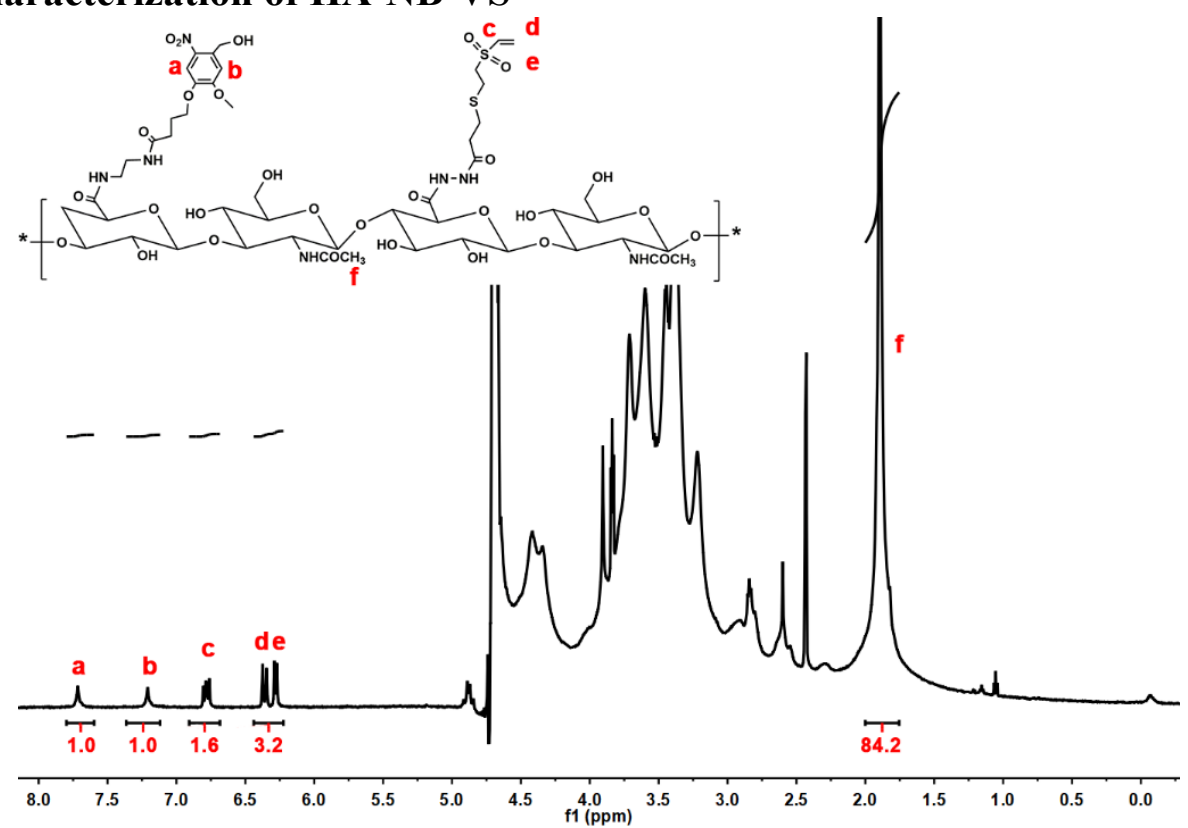

Figure S3. ${ }^{1} \mathrm{H}$ NMR spectrum $\left(600 \mathrm{MHz}, \mathrm{D}_{2} \mathrm{O}\right)$ of $\mathbf{H A}-\mathbf{N B}-\mathbf{V S}$.

The substitution degree was calculated by the NMR integral analysis using the following formula $\mathrm{Y} \%=\mathrm{I}_{[\mathrm{c}]} /\left(\mathrm{I}_{[\mathrm{f}]} \div 3\right) \times 100 \%$.

\section{In situ formation of HA-MMP hydrogel via thiol-Michael reaction}



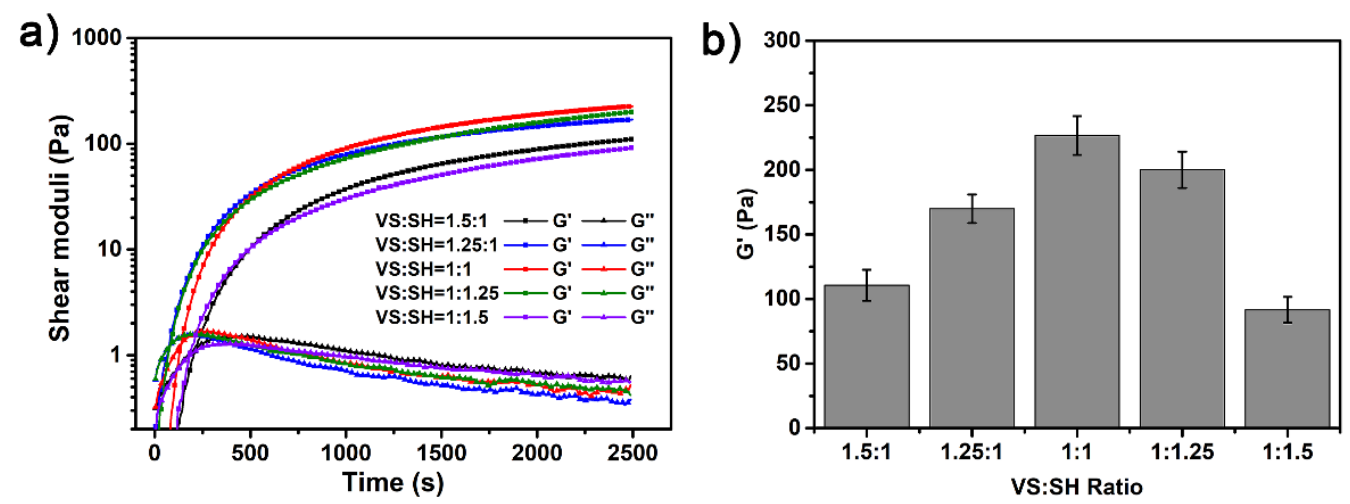

Figure S4. a-b) In situ rheological analysis for gel formation and the effects of hydrogel formulation on final moduli. For data in b), four sets of independent samples were monitored.

\section{NB photolysis and its ability to conjugate protein in hydrogel}

\subsection{The stability of imine-bond in NB-3 generated from PAAL}
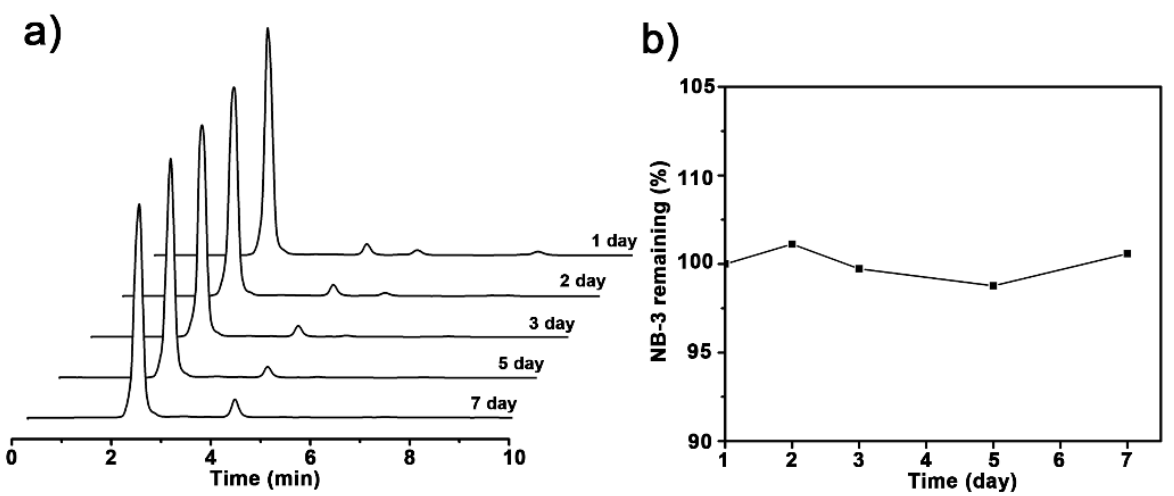

Figure S5. a) The evolution of HPLC spectra of NB-3 kept in acetonitrile-water solution (4/1, v/v) over time. b) The calculated normalized remaining ratio of NB-3 from a).

\subsection{Photolysis of HA-NB and HA-MMP hydrogel}
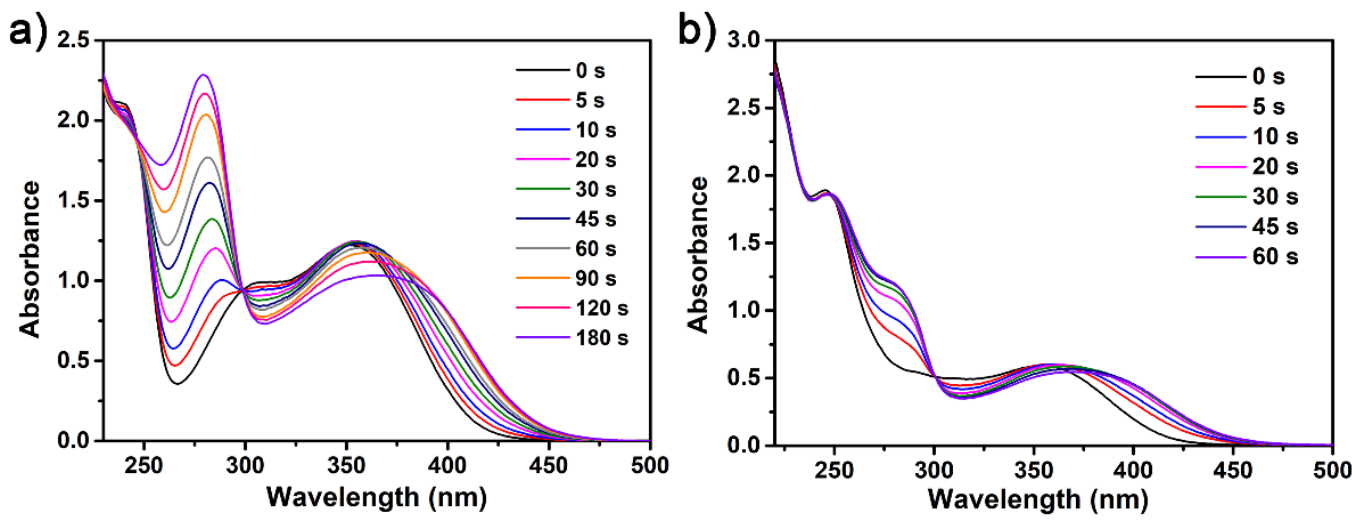
Figure S6. UV-vis spectra evolution for the photolysis of HA-NB $\left(2 \mathrm{mg} \mathrm{mL}^{-1}\right)$ in PBS a) and HA-MMP hydrogel film b) upon $365 \mathrm{~nm}$ LED light irradiation $\left(10 \mathrm{~mW} \mathrm{~cm}^{-2}\right)$.

\subsection{FTIR-ATR spectra for protein photoimmobilization in hydrogel}

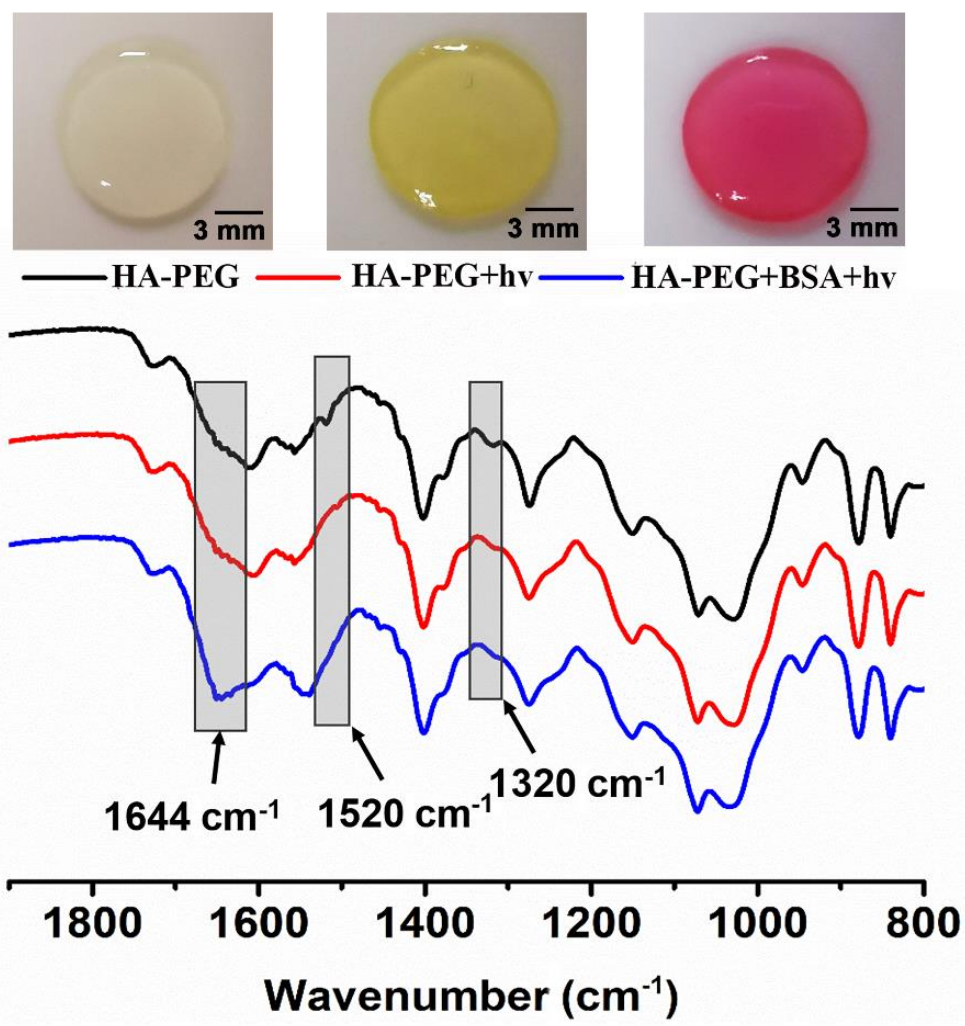

Figure S7. The ATR-FTIR spectra for photolysis analysis and BSA immobilization in HA-PEG. Up images are the hydrogels of HA-PEG, HA-PEG+hv and HA-PEG +BSA-Rho+hv (BSA-Rho was used for better visual).

"HA-PEG+hv" means the xerogel from the irradiated HA-PEG hydrogel, the disappeared peak at $1320 \mathrm{~cm}^{-1}$ and $1520 \mathrm{~cm}^{-1}\left(-\mathrm{NO}_{2}\right.$ stretching vibration) confirmed the photolysis of the NB group.

"HA-PEG+BSA + hv" means the xerogel from the irradiated HA-PEG hydrogel with BSA-Rho premix, the increased peak at $1644 \mathrm{~cm}^{-1}$ (amide stretching vibration of BSA) confirmed the BSA immobilization in hydrogel.

\section{Photopatterning of protein and cells}

\subsection{D photopatterning of BSA-Rho}

BSA-Rho and BSA-FITC were used as protein models in the assay and prepared as reported. ${ }^{1}$ 
a)

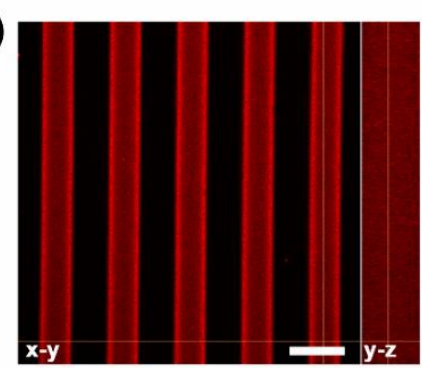

b)

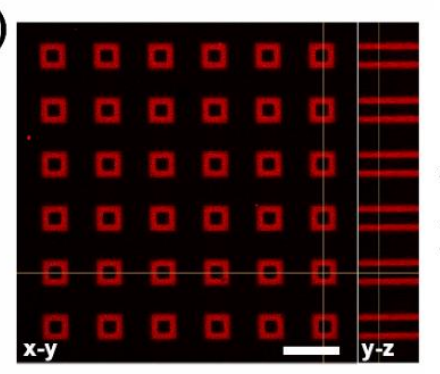

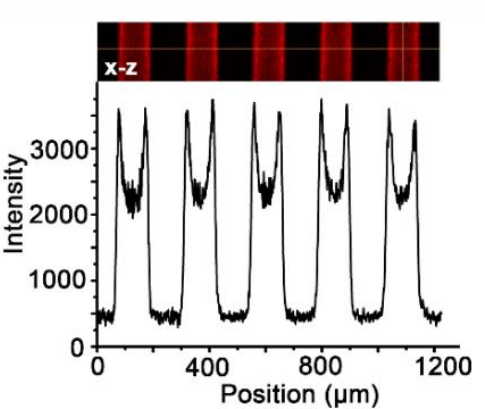
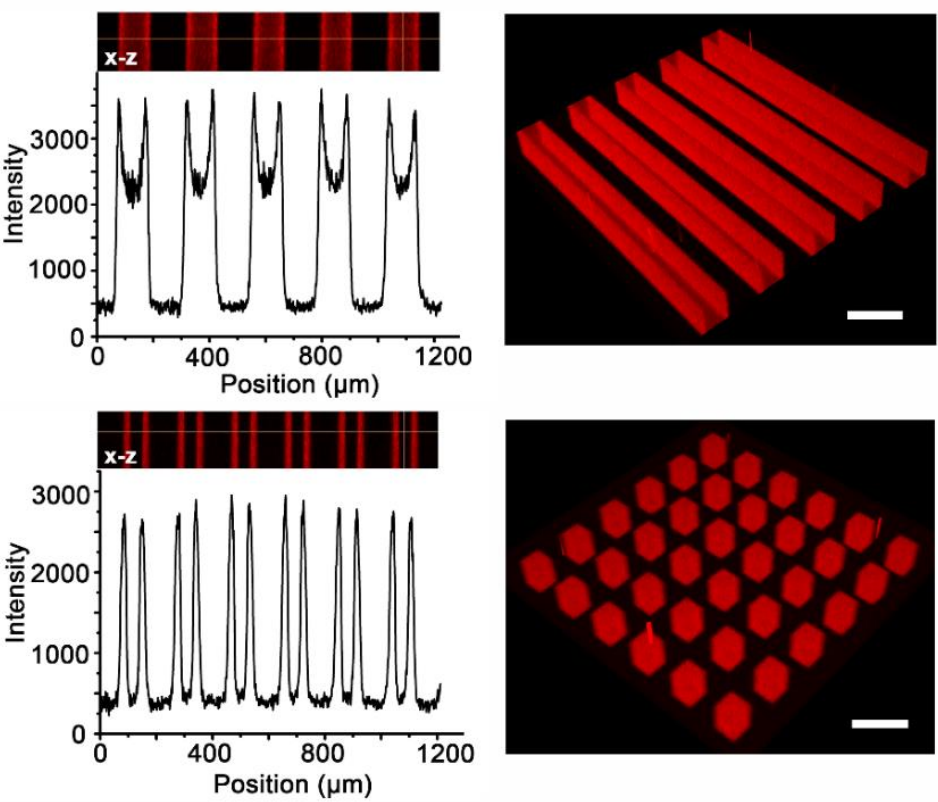

Figure S8. BSA-Rho 3D patterning in HA-MMP hydrogels by photomasks after 3 min irradiation $\left(10 \mathrm{~mW} \mathrm{~cm}^{-2}\right)$ : a) stripes with $200 \mu \mathrm{m}$ width and spacing and b) frames with $100 \mu \mathrm{m}$ extra and $50 \mu \mathrm{m}$ intra side-length. Images represent $100 \mu \mathrm{m}$ depth at $\mathrm{z}$ projection, scale bars are $200 \mu \mathrm{m}$. The protein concentration was fixed at $10 \mu \mathrm{M}$.

\subsection{Sequential photopatterning of BSA-FITC and BSA-Rho in HA-MMP hydrogel}

First, HA-MMP hydrogels were soaked in BSA-Rho solution $(10 \mu \mathrm{M})$ and patterned by photomask with strip lines ( $365 \mathrm{~nm}$ LED light source, $10 \mathrm{~mW} \mathrm{~cm}{ }^{-2}$ for 3 mins). After washing in PBS overnight, the hydrogel was resoaked in BSA-FITC solution $(10 \mu \mathrm{M})$ and followed by photopatterning with the mask in perpendicular way and washing process. The patterned hydrogel was imaged by using A1R Nikon confocal microscope from two channels, rhodamine channel $\lambda_{\mathrm{ex}}=561 \mathrm{~nm}$ (red) and FITC channel $\lambda_{\mathrm{ex}}=488 \mathrm{~nm}$ (green). 

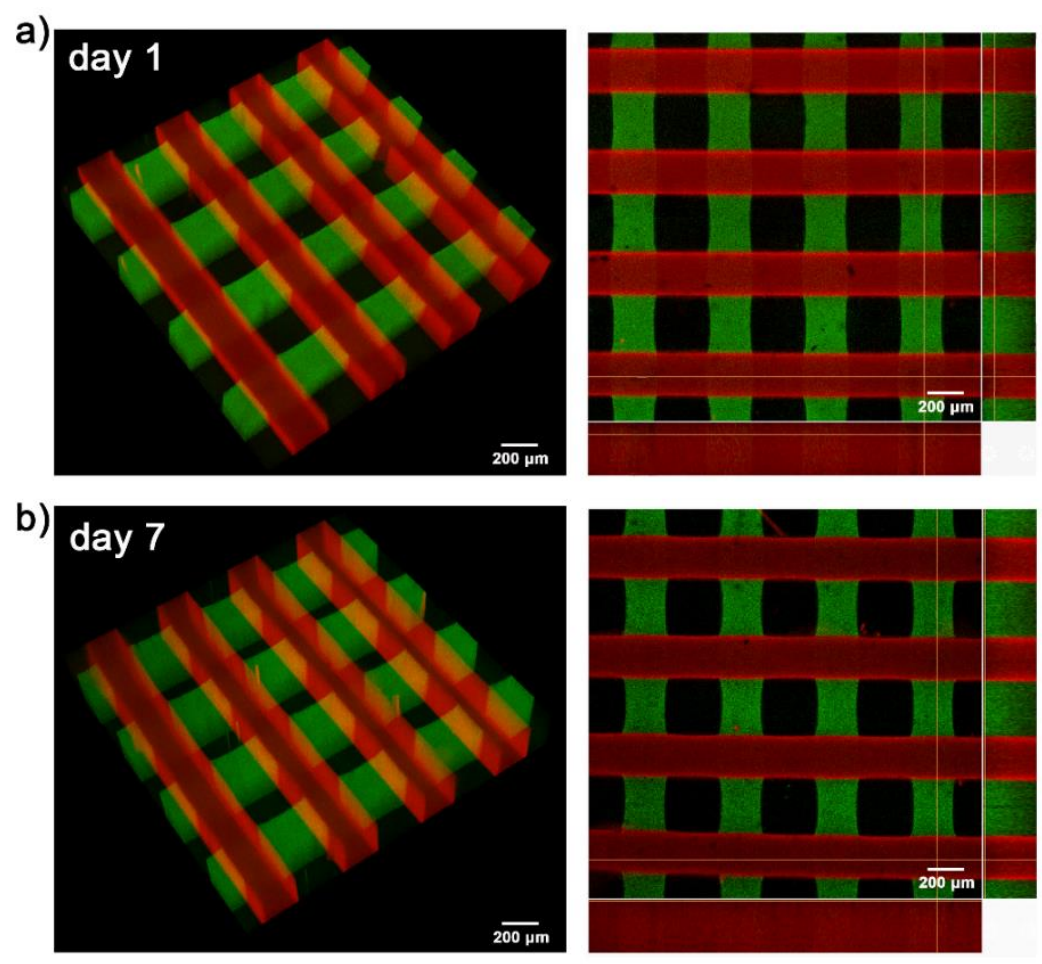

Figure S9. 3D and 2D confocal images for sequential protein patterns of BSA-Rho and BSA-FITC in HA-MMP hydrogel after soaking in PBS for a) 1 day and b) 7 days.

\subsection{Cell adhesion on hydrogel mediated by peptide or protein patterning}



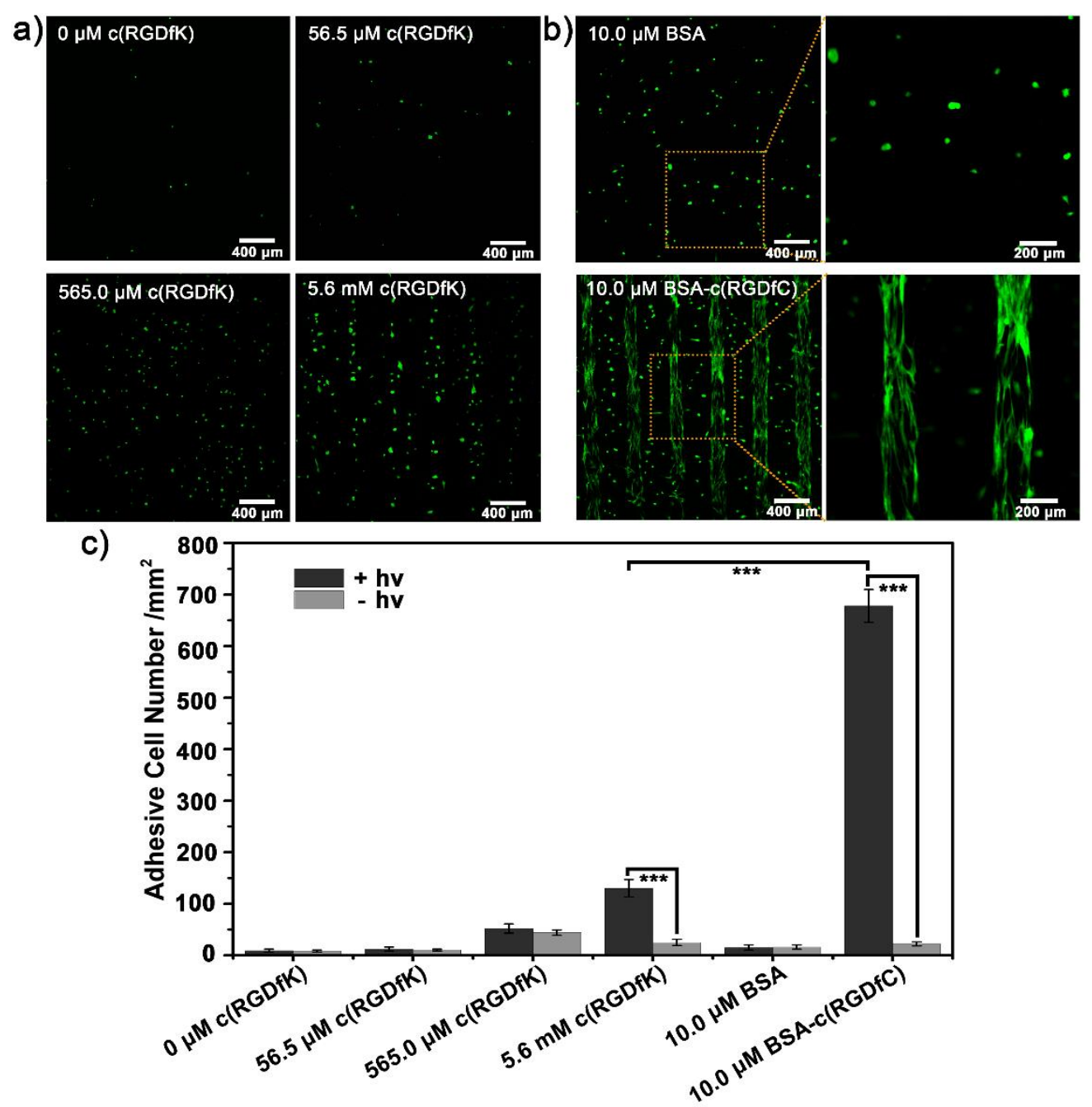

Figure S10. Confocal micrographs (Calcein channel, $\lambda_{\mathrm{ex}}=488 \mathrm{~nm}$ ) and quantifications of HDF cells numbers on patterned HA-MMP hydrogels. a) photopatterning of $\mathrm{c}(\mathrm{RGDfK})$ at different concentrations, b) photopatterning of BSA and BSA-c(RGDfC). c) Data representation of the adhered cell densities in a) and b). "+hv" means cells in the irradiated areas, "-hv" means cells in the nonirradiated areas. A total of $5 \times 10^{4}$ cells were seeded on the hydrogels and co-cultured for $12 \mathrm{~h}$.*** denotes $\mathrm{p}<0.001$.

\subsection{Synthesis and determination of the substituted degree of BSA-c(RGDfC)}

The synthesis procedure of BSA-c(RGDfC) was performed as previously described. ${ }^{2}$ Briefly, $50 \mathrm{mg}$ BSA $(0.75 \mu \mathrm{mol})$ was dissolved in $4 \mathrm{~mL}$ PBS $(100 \mathrm{mM}$, pH $=7.2)$ and then activated by dropping sulfo-succinimidyl 4-(N-maleimidomethyl) cyclohexane-1-carboxylate (sulfo-SMCC, $5.0 \mathrm{mg}$ in $1 \mathrm{~mL}$ warm PBS). After stirring for $1 \mathrm{~h}$ at room temperature, the solution was desalted with a Sephadex G-50 column for 1 
$\mathrm{h}$ and incubated with cyclo(Arg-Gly-Asp-d-Phe-Cys) (c(RGDfC), $1.0 \mathrm{mg} \mathrm{mL}{ }^{-1}$ ) overnight at room temperature. Then, the mixture was transferred to a dialysis bag (MWCO 3500, Spectrum ${ }^{\circledR}$ ) and dialyzed against deionized water at $4{ }^{\circ} \mathrm{C}$ for $3 \mathrm{~d}$. The newly purified protein solution was freeze-dried and stored at $-20{ }^{\circ} \mathrm{C}$ prior to use. MALDI-TOF and SDS-PAGE analyses were conducted to characterize the modified protein BSA-c(RGDfC), and the substitution degree of $\mathrm{c}(\mathrm{RGDfC})$ was determined by MALDI-TOF.

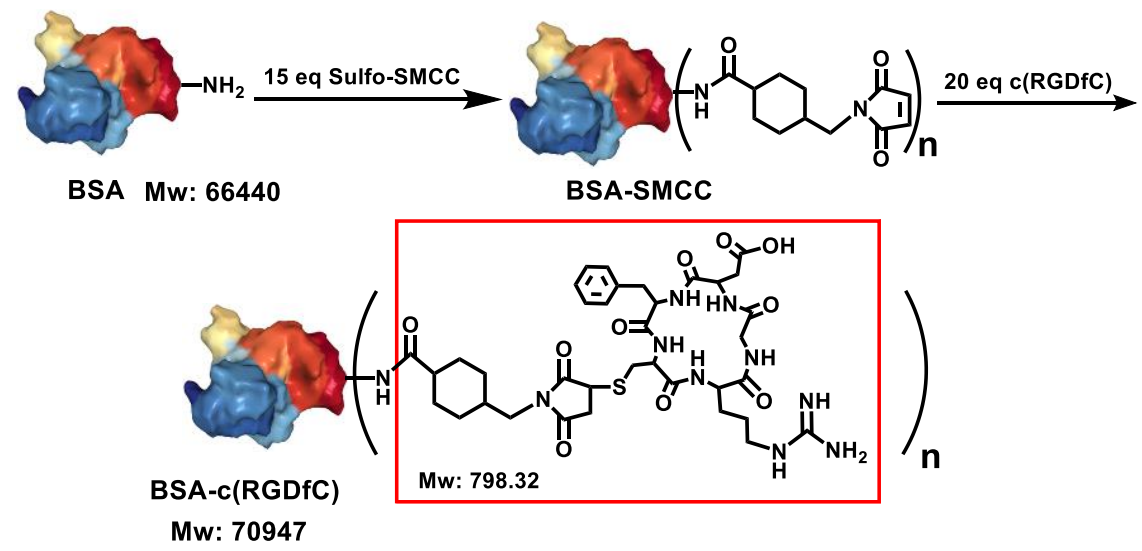

Figure S11. The synthetic procedure for BSA-c(RGDfC). Structure of BSA from PDB 4F5S.

From above procedure, the number of substituents (n) can be determined using following equation:

$M w_{B S A-C(R G D F C}=M w_{B S A}+797.32^{*} n$

Based on the molecular weight from the mass spectra, $\mathrm{n}$ can be determined as 5.65, then the substitution degree can be determined as $9.4 \%$ (relative to 60 amine groups in a BSA molecule).

\subsection{The effects of the photoirradiation of hydrogel on cell adhesion}




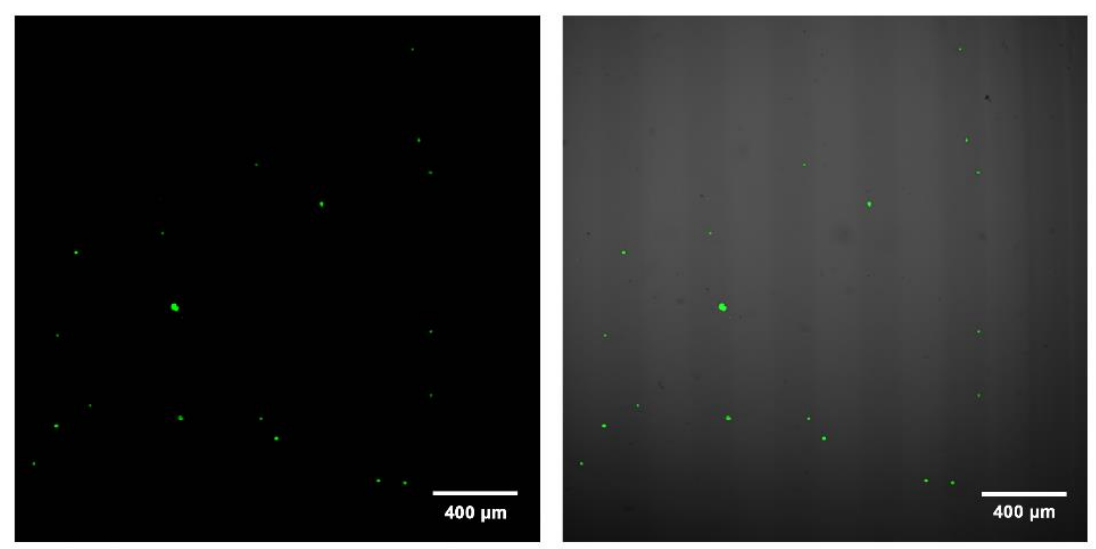

Figure S12. Confocal images of cells adhesion on photoirradiated HA-MMP hydrogels. A total of $5 \times 10^{4}$ cells were seeded on the hydrogels and photoirradiated for $3 \min (365$ $\mathrm{nm}$ LED, $10 \mathrm{~mW} \mathrm{~cm}^{-2}$ ) and co-cultured for $12 \mathrm{~h}$.

\section{AND logic gate for 3D cell culture}

\subsection{Light cytotoxicity for the AND logic gate manipulation}

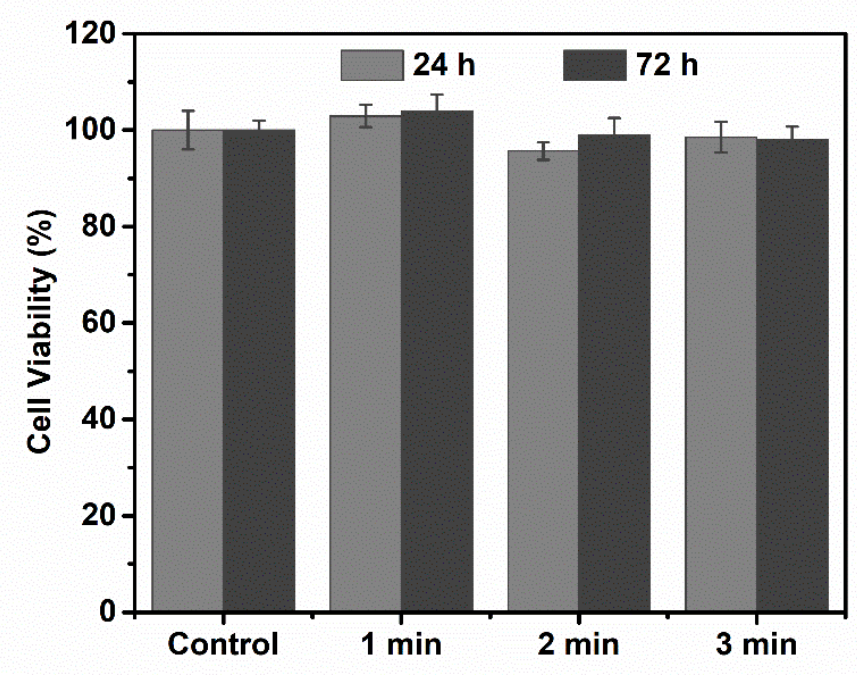

Figure S13. The cytotoxicity of irradiation by $365 \mathrm{~nm}$ LED light.

\subsection{The longitudinal depth of photoimmobilization protein in hydrogel.}

Given that light attenuation would lead to protein longitudinal gradient in hydrogel, BSA-Rho was used as the model to explore the depth of photopatterning. The irradiation condition was same as above ( $365 \mathrm{~nm}$ LED light source, $10 \mathrm{~mW} \mathrm{~cm}{ }^{-2}$ for 3 mins) by using a square-shaped photomask. 
a)

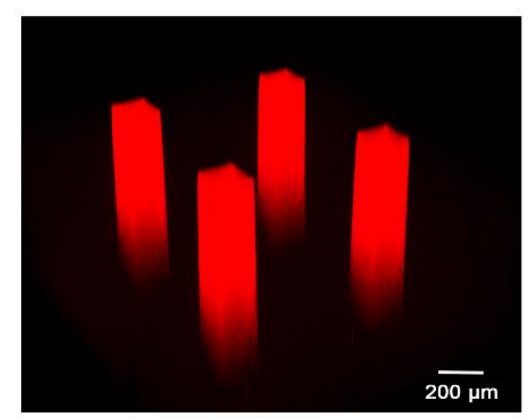

b)

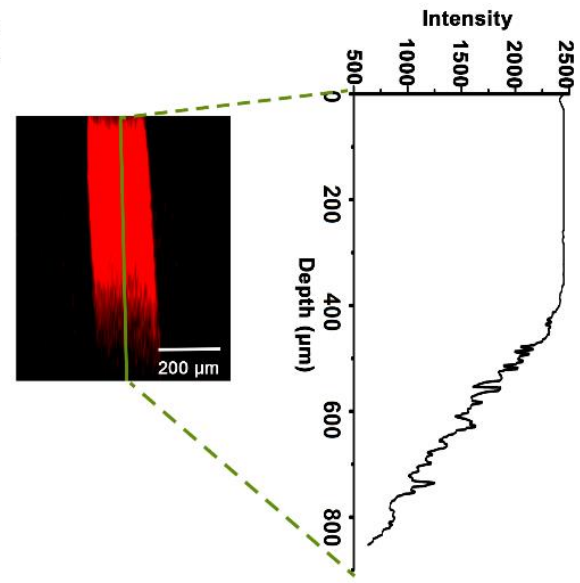

Figure S14. a) A representative image of the BSA-Rho 3D photopatterning constructed from a series of XY corss-senctional micrographs over a $0.85 \mathrm{~mm}$ depth. b) The longitudinal fluorescence intensity profile along the central axis of the square.

\subsection{AND logic gate for cell viability and spreading}
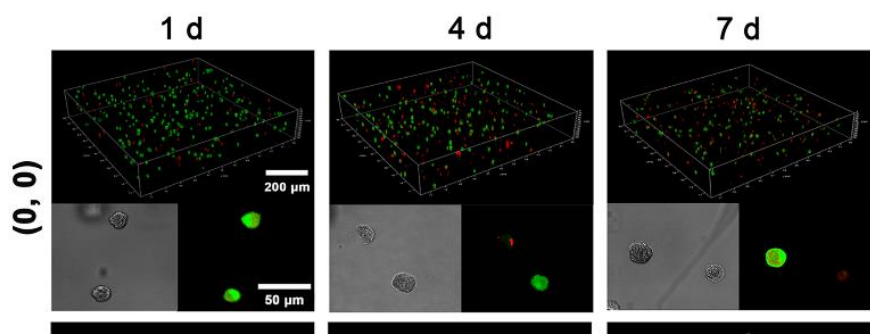

$12 d$
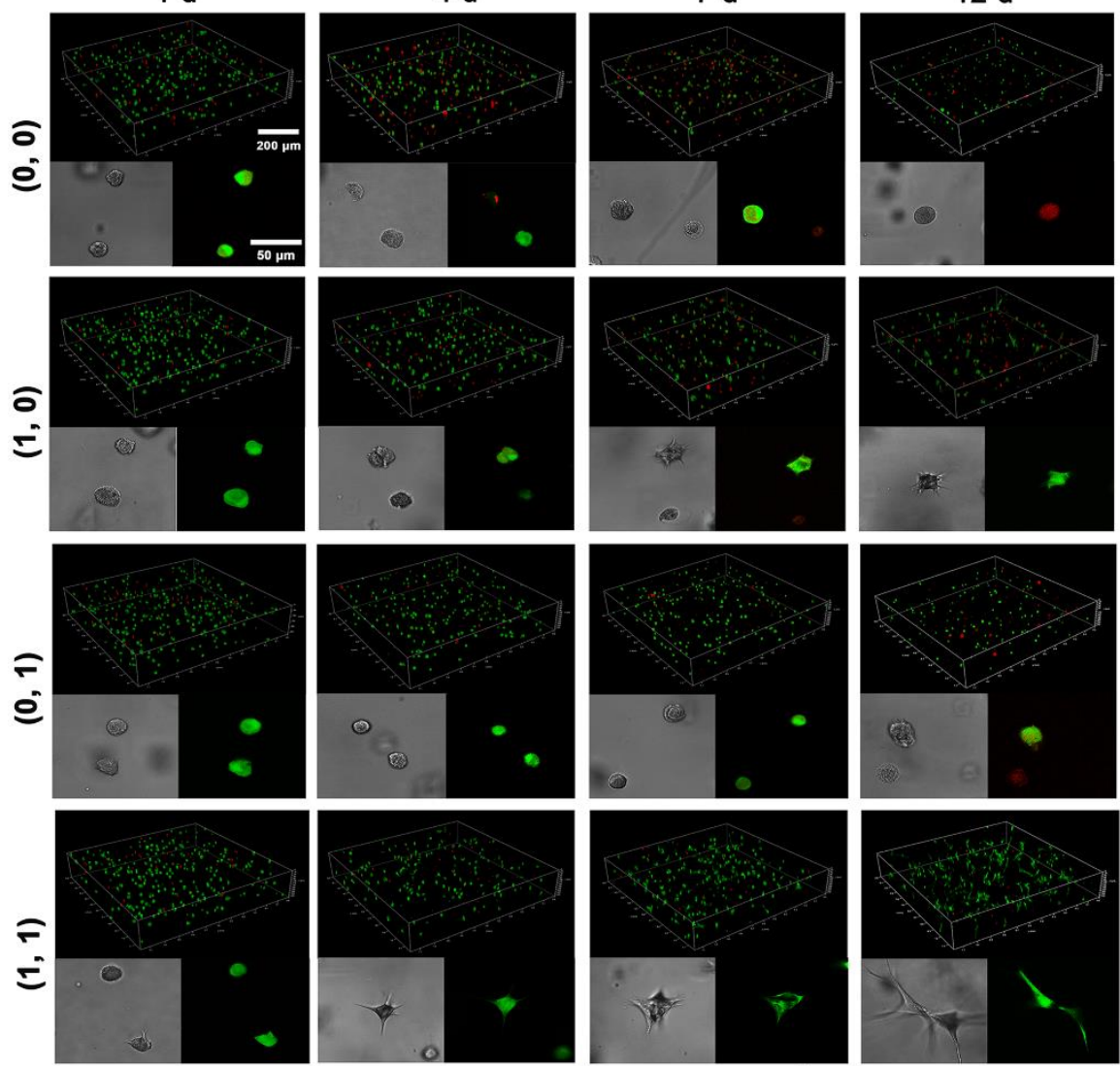

Figure S15. Confocal images of cells in the user-programed hydrogels at day 1, 4, 7 and 12. (0, 0) means BSA+HA-PEG hydrogel, (1, 0) means BSA-c(RGDfC)+HA- 
PEG hydrogel, $(0,1)$ means BSA+HA-MMP hydrogel, and $(1,1)$ means BSAc(RGDfC)+HA-MMP hydrogel.

6.4 AND logic gate for 3D cell migration

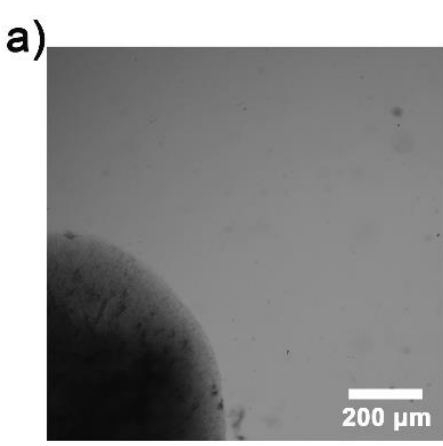

$(0,0)$

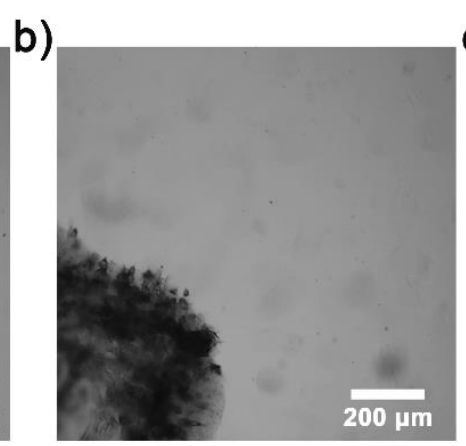

$(1,0)$

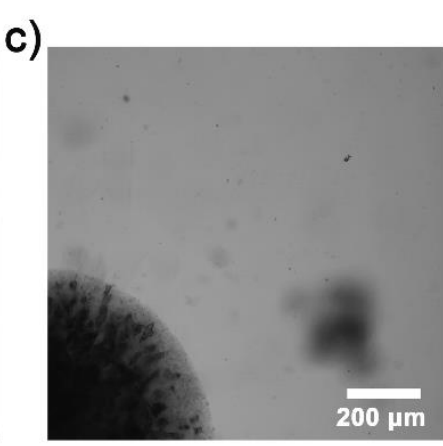

$(0,1)$

Figure S16. The microscopic images for HDF cells migration in hydrogels after $12 \mathrm{~d}$ culture: a) BSA+HA-PEG hydrogel, b) BSA-c(RGDfC)+HA-PEG hydrogel, and c) BSA+HA-MMP hydrogel.

\section{Photo-modulated 3D cell culture and migration in HA-MMP}

\section{hydrogels}

a)

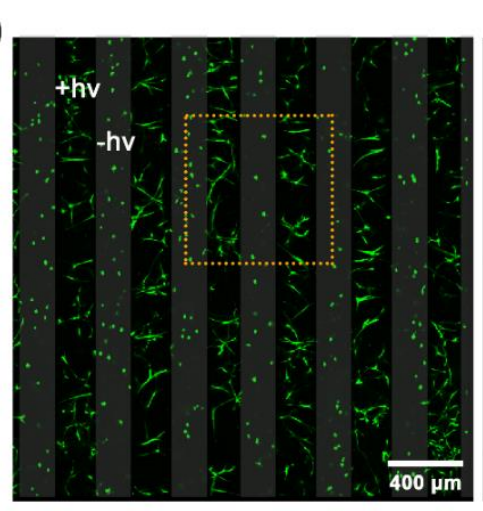

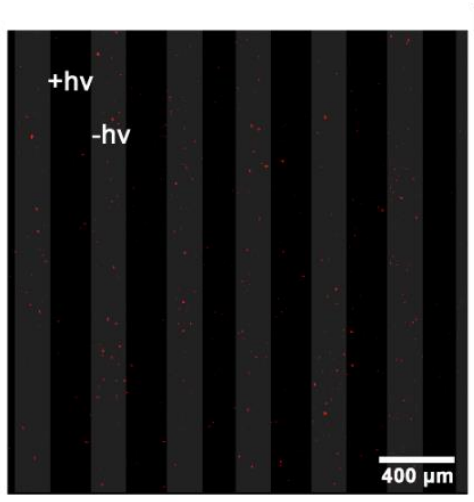

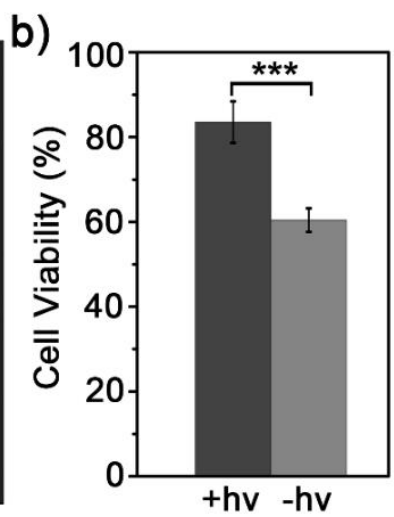

Figure S17. Photo-modulated 3D HDF cell culture and migration in BSA(RGDfC)+HA-MMP hydrogels. a) Z-stacked confocal images (200 $\mu \mathrm{m}$ depth) of encapsulated cells stained by a live/dead assay after $12 \mathrm{~d}$ of culture. b) Evaluation of cell viability in photopatterned hydrogels. ${ }^{* * *}$ denotes $\mathrm{p}<0.001$. 

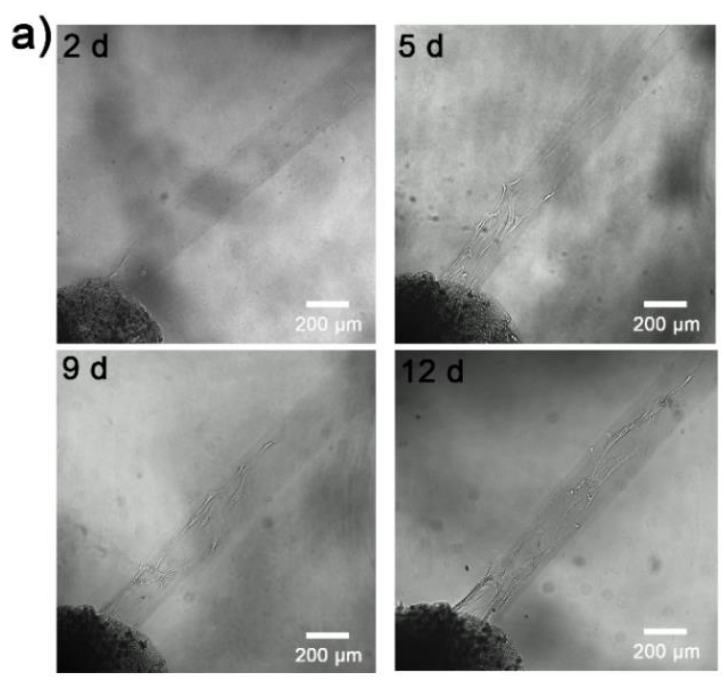

b)

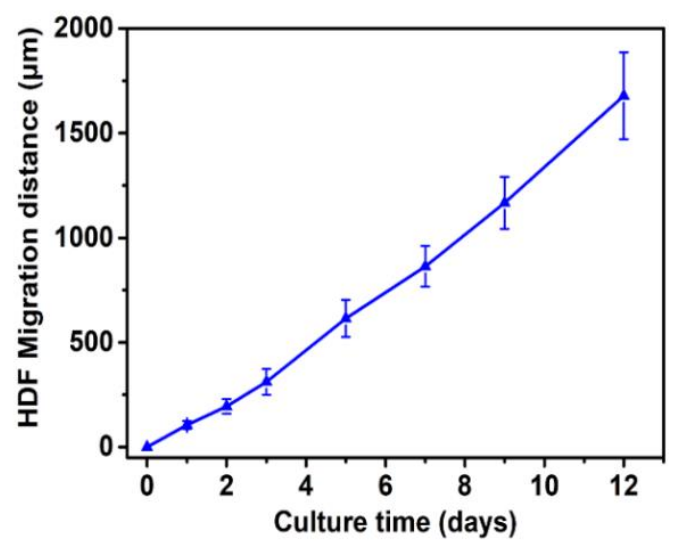

Figure S18. Time dependent analysis of a) microscopic images and b) cell migration distance for HDF cells in patterned BSA-c(RGDfC)+HA-MMP hydrogels.

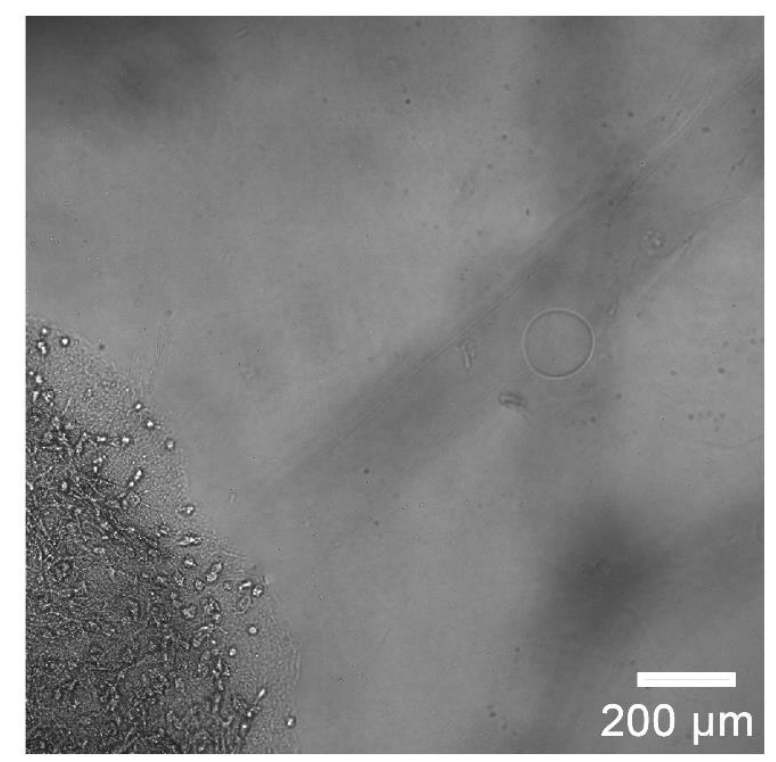

Figure S19. The bright field image for cell migration in BSA patterned hydrogels after $12 \mathrm{~d}$ of culture.

\section{References}

1) Maddala, S. P.; Mastroianni, G.; Velluto, D.; Sullivan, A. C. Intracellular delivery of BSA by phosphonate@silica nanoparticels, J. Mater. Chem. B 2015, 3, 6057-6070.

2) Chen, Y.; Dai, X.; Huang, L.; Sun, Y.; Chan, H. N.; Shen, B.; Zeng, X.; Wu, Z.; Hsing, I. M.; Guo, Z.; Wu, H. A Universal and Facile Approach for the Formation of a Protein Hydrogel for 3D Cell Encapsulation. Adv. Funct. Mater. 2015, 25, 6189-6198. 